\title{
AIP
}

\section{A revised diagrammatic technique for the degenerate Anderson model}

T. K. Lee and F. C. Zhang

Citation: Journal of Applied Physics 55, 1936 (1984); doi: 10.1063/1.333524

View online: http://dx.doi.org/10.1063/1.333524

View Table of Contents: http://scitation.aip.org/content/aip/journal/jap/55/6?ver=pdfcov

Published by the AIP Publishing

\section{Articles you may be interested in}

A diagrammatic solution of the single-impurity Anderson model in the Kondo regime

AIP Conf. Proc. 918, 272 (2007); 10.1063/1.2751994

Abstract: Hydrogen and transition metal adatom chemisorption on transition metals as a function of $d$ bandfilling:

Tenfold degenerate Hubbard and Anderson models

J. Vac. Sci. Technol. 13, 350 (1976); 10.1116/1.568877

Diagrammatic Approach to the Anderson Model for Dilute Alloys

J. Appl. Phys. 42, 1460 (1971); 10.1063/1.1660293

Diagrammatic Technique for Constructing Matrix Elements

J. Math. Phys. 11, 3420 (1970); 10.1063/1.1665143

Charge Redistribution in the Anderson Model

Am. J. Phys. 38, 996 (1970); 10.1119/1.1976555

MIT LINCOLN

LABORATORY CAREERS

Discover the satisfaction of innovation and service

to the nation
- Space Control

- Air \& Missile Defense

- Communications Systems \& Cyber Security

- Intelligence, Surveillance and Reconnaissance Systems

- Advanced
Electronics
- Tactical Systems
- Homeland
Protection
- Air Traffic Control

- Air Traffic Control

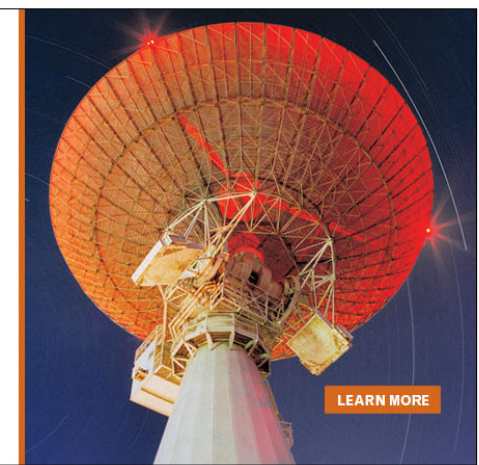




\section{A revised diagrammatic technique for the degenerate Anderson model}

T. K. Lee

Department of Physics, Virginia Polytechnic Institute and State University, Blacksburg, Virginia 24061

F. C. Zhang

School of Physics and Astronomy, University of Minnesota, Minneapolis. Minnesota 55455

The Goldstone diagrammatic technique developed by Keiter and Kimball for single impurity Anderson model is reformulated. Instead of having the self-energy functions defined on the real axis as the Brillouin-Wigner theory, we have defined the functions on the complex plane. This avoids the complicated and cumbersome regularization procedure required in the Keiter and Kimball formulation. Most important of all it makes the numerical calculations possible. The exact partition function may be written down in terms of irreducible self-energy diagrams. The Green function and spectral function are derived.

PACS numbers: $75.20 . \mathrm{Hr}$

Recently, there has been much interest in mixed-valence compounds. ${ }^{1,2}$ It is generally believed that the degenerate Anderson Hamiltonian ${ }^{3,4}$ is the simplest microscopic model which may describe the mixed-valence system ${ }^{5}$ for a single rare-earth ion or a periodic lattice of ions.

For the system of a single mixed-valence impurity, there are some exact results obtained by Bethe Ansatz meth$\mathrm{od}^{6,7}$ and numerical renormalization group technique. ${ }^{8}$ But a lot of theoretical efforts are devoted to find a suitable perturbative method so that it can be applied to systems with a lattice of mixed-valence ions.

The standard perturbation theory for the model was devised by Keiter and Kimball ${ }^{9}$ more than ten years ago. By using Goldstone diagrams, they showed that the physics of a single impurity mixed-valence system can be described in terms of the statistical quasiparticles. ${ }^{10}$ The energies of the quasiparticles are determined from the self-consistent Brillouin-Wigner type equations. Based on their theory, thermodynamic properties of the model are studied ${ }^{1 t-13}$ in the mixed-valence regime.

However, there are practical difficulties in Keiter and Kimball theory. To avoid accidentally vanishing energy denominators in the Brillouin-Wigner equations, a very complicated regularization procedure must be adopted. ${ }^{9.14} \mathrm{We}$ may overcome this difficulty only at zero temperature ${ }^{13}$ or for low order diagrams. " functions are also difficult to calculate. ${ }^{14,15}$

In this paper we present a new diagrammatic theory, in which Goldstone diagrams are evaluated in the complex plane excluding the real axis. Physical quantities are related to the real and imaginary parts of the contributions from the diagrams. Thus there is no regularization needed. Higher order diagrams can be calculated straightforwardly. Green functions and the spectral function are also easily formulated.

The degenerate Anderson model ${ }^{3,4}$ for a single rareearth impurity is given by the Hamiltonian

$$
\begin{aligned}
H= & \sum_{k \sigma} \epsilon_{k \sigma} C_{k \sigma}^{+} C_{k \sigma}+\sum_{m} \epsilon_{j m} X_{m m} \\
& +\sum_{k \sigma m} V_{k \sigma m} C_{k \sigma}^{+} X_{m}+\text { h.c. },
\end{aligned}
$$

which describes a one-electron transition between a local configuration of the rare-earth impurity and the conduction band states which are described by the annihilation operators $C_{k \sigma} . \epsilon_{f m}$ is the energy separation between the configuration $\mid m>$ [with $n-14 f$ electrons in states with quantum number $(J, m)]$ and the configuration $\mid 0>$ (with $n 4 f$ electrons in state $J=0)$. The projection operator $X_{m}$ $=|0\rangle\langle m|$ changes the rare-earth impurity from configurations $|m\rangle$ to $|0\rangle$, and the operator $X_{m m}=X_{m}{ }_{m} X_{m}$ $=|m><m|$. There are $N=2 J+1$ degenerate configurations $\mid m>$.

A perturbative diagrammatic approach of treating the Hamiltonian has been devised by Keiter and Kimball. ${ }^{9}$

The hybridization interaction in Eq. (1) is treated as a perturbation. Because the Hubbard operators $X_{m}$ and $X_{m}^{+}$ do not satisfy the usual anticommutation relation, Keiter and Kimball devised a time ordered Goldstone diagrammatic technique. Readers please refer to Ref. 9 for details of diagrammatic rules.

The partition function can be obtained from these diagrams":

$$
\begin{aligned}
Z / Z_{0}= & 1+\sum_{i=1}^{\infty} \sum_{\substack{\text { all } 2 l \text { th } \\
\text { order diagrams }}} \sum_{\left.\mid k_{l}\right\}}(-)^{c} n V F \\
& \times \frac{1}{2 \pi i} \int d z e^{-\beta z} z^{-1} \prod_{i=1}^{2 l}\left(z-\epsilon_{i}\right)^{-1},
\end{aligned}
$$

where summation with $\left\{k_{l}\right\}$ means to sum over all possible $k_{i}, \sigma_{i}$, and $m_{i}, i$ runs from 1 to $l$, and $n$ denotes the initial occupation probability, $n_{0}$ and $n_{m}$ for states $|0\rangle$ and $|m\rangle$, respectively. $V$ stands for the product of matrix elements $\left|V_{k_{1} \sigma_{1} m_{1}}\right|^{2}\left|V_{k_{2} \sigma_{2} m_{2}}\right|^{2} \ldots . .\left|V_{k_{1} \sigma_{l} m_{l}}\right|^{2}$, and $\boldsymbol{F}$ for the product of statistical factors .... $f_{k \sigma}\left(1-f_{k^{\prime} \sigma} \cdot\right)^{\prime}$, , superindex $C$ is the number of crossings of $k$-electron lines, $\epsilon_{i}$ are the excitation energies which can be read from the diagrams, $\epsilon_{i}=-\sum_{j=1}^{i} \Delta E_{j}$. And $\Delta E_{j}$ is the sum of the $k$-electron energy $\epsilon_{k \sigma}$ (with a positive sign, if the arrow on the full line labeled $k \sigma$ points away from the dot at the time, and with a negative sign if the arrow points towards the dot) and the $f$-electron energy $\epsilon_{f m}$ (with the same sign rule). The contour of integration encircles all poles of the integrand. $Z_{0}$ is the partition function for $H_{0}$. Starting with Eq. (2), Keiter and Kimball developed a statistical quasiparticle theory.

Here we shall present a new approach. We group the 
diagrams in terms of the initial states $\mid 0>$ and $\mid m>$. Equation (2) can be written as

$$
\begin{aligned}
Z / Z_{0}= & \frac{n_{0}}{2 \pi i} \int d z \frac{e^{-\beta z}}{z} \\
& \times\left\{1+\sum_{i=1} \sum_{\mid 0>} \sum_{\left\{k_{l}\right\}} \frac{(-1)^{c} V F}{\prod_{i=1}^{2 l}\left(z-\epsilon_{i}\right)}\right\} \\
& +\sum_{m} \frac{n_{m}}{2 \pi i} \int d z \frac{e^{-\beta z}}{z} \\
& \times\left\{1+\sum_{l=1} \sum_{\mid m>} \sum_{\left\{k_{l}\right\}} \frac{(-1)^{c} V F}{\prod_{i=1}^{2 l}\left(z-\epsilon_{i}\right)}\right\},
\end{aligned}
$$

where the summations with indices $\mid 0>$ and $\mid m>$ indicate summations over all diagrams with initial states $\mid 0>$ and $|m\rangle$, respectively. Furthermore, we group the diagrams as reducible or irreducible ones. A reducible diagram can be separated into more than one irreducible part. Hence the contributions to the sum from the reducible diagrams of Eq. (3) can be expressed as the products of the contributions from the irreducible diagrams. The first sum in Eq. (3) is

$$
\sum_{l=1}^{\infty} \sum_{\mid 0_{>}} \sum_{\left|k_{t}\right|} \frac{(-1)^{c} V F}{\prod_{i=1}^{2 l}\left(z-\epsilon_{i}\right)}=\sum_{i=1}^{\infty}\left(\sum_{i r \mid 0>} \sum_{\left|k_{i}\right\rangle} \frac{(-1)^{c} V F}{\prod_{i=1}^{2 j}\left(z-\epsilon_{i}\right)}\right)^{\prime}
$$

where the second summation in the right-hand side indicates the sum over all irreducible diagrams with initial states $|0\rangle$.

Noting that $\epsilon_{2 j}=0$ in Eq. (4), we define a self-energy function given by

$$
S(z)=\sum_{i r \mid 0>} \sum_{\left|k_{\ell}\right|} \frac{(-1)^{c} V F}{\prod_{i=1}^{2 j-1}\left(z-\epsilon_{i}\right)} .
$$

Substituting Eqs. (5) and (4) into the first contour integration in Eq. (3), we obtain

$$
\frac{n_{0}}{2 \pi i} \int d z e^{-\beta z}[z-S(z)]^{-1} \text {. }
$$

The same strategy can be applied to the second term in Eq. (3). Therefore

$$
\begin{aligned}
Z / Z_{0}= & \frac{n_{0}}{2 \pi i} \int d z \frac{e^{-\beta z}}{z-S(z)} \\
& +\sum_{m} \frac{n_{m}}{2 \pi i} \int d z \frac{e^{-\beta z}}{z-T_{m}(z)}
\end{aligned}
$$

The self-energy functions $S$ and $T_{m}$ are the same as those obtained by Keiter and Kimball, ,,13 except that these functions are now defined in the complex plane. Because the integrands in Eq. (6) are analytic in the whole complex plane apart from the real axis, we may change the contour of the integrations continuously and obtain

$$
\begin{aligned}
Z / Z_{0}= & \frac{n_{0}}{\pi} \int_{-\infty}^{\infty} d x \operatorname{Im} \frac{e^{-\beta \mathrm{x}}}{x-i \delta-S(x-i \delta)} \\
& +\sum_{m} \frac{n_{m}}{\pi} \int_{-\infty}^{\infty} d x \operatorname{Im} \frac{e^{-\beta x}}{x-i \delta-T_{m}(x-i \delta)}
\end{aligned}
$$

where $\delta$ is an infinitesimal positive number.

We may rewrite the partition function in the form

$$
Z /\left(Z_{0}\right)_{c}=\int_{-\infty}^{\infty} e^{-\beta x} g^{*}(x) d x,
$$

where the effective density of states is given by

$$
\begin{aligned}
g^{*}(x)= & \frac{1}{\pi}\left\{\operatorname{Im}[x-i \delta-S(x-i \delta)]^{-1}\right. \\
& \left.+\sum_{m} \operatorname{Im}\left[x-\epsilon_{f m}-i \delta-T_{m}\left(x-\epsilon_{f m}-i \delta\right)\right]^{-1}\right\},
\end{aligned}
$$

which is temperature dependent.

From Eq. (9) we can see that the peaks of the effective density of states appear at the points which are the solutions of the equations

$$
\begin{aligned}
& \chi-\operatorname{Re} S(x-i \delta)=0, \\
& \chi-\epsilon_{f m}-\operatorname{Re} T_{m}\left(x-\epsilon_{f m}-i \delta\right)=0,
\end{aligned}
$$

which correspond to the local $f$-hole and $f$-electron, respectively. The width of a peak is proportional to $\operatorname{Im} S\left(x_{0}-i \delta\right)$ or $\operatorname{Im} T_{m}\left(x_{m}-\epsilon_{f m}-i \delta\right), x_{0}$ and $x_{m}$ being the solutions of Eqs. (11) and (10), respectively. Of course, the width relates directly to the lifetime. Multiple roots of Eq. (10) and/or Eq. (11) mean multiple peaks.

For $S$ and $T$, we have the identities

$$
\begin{aligned}
& \frac{1}{\pi} \int d x \operatorname{Im}[x-i \delta-S(x-i \delta)]^{-1}=1, \\
& \frac{1}{\pi} \int d x \operatorname{Im}\left[x-i \delta-T_{m}(x-i \delta)\right]^{-1}=1 .
\end{aligned}
$$

If bandwidth $D$ is much larger than the other parameters such as $\left|\epsilon_{f m}\right|$ and $N \Delta$, and if $N$ is large, we have shown in a previous paper ${ }^{13}$ that the diagrams with crossing full lines can be neglected. In that case, functions $S$ and $T$ are related through the following integral equations:

$$
\begin{aligned}
S(z)= & \int d \epsilon f(\epsilon)\left[z+\epsilon-\epsilon_{f}-T\left(z+\epsilon-\epsilon_{f}\right)\right]^{-1}, \\
T(z)= & \frac{1}{N} \int d \epsilon[1-f(\epsilon)] \\
& \times\left[z-\epsilon+\epsilon_{f}-S\left(z-\epsilon+\epsilon_{f}\right)\right]^{-1} .
\end{aligned}
$$

Throughout this paper we shall choose the unit $N \Delta=1$, where

$$
N \Delta=\sum_{k \sigma m}\left|V_{k \sigma m}\right|^{2} \delta\left(w-\epsilon_{k \sigma}\right)
$$

For large $D$, the integral equations can be solved analytically for $N=1$ at $T=0{ }^{\circ} \mathrm{K} .{ }^{16}$ The results are in very good agreement with the exact solution including cross terms. For $N$ much larger than 1 , the cross terms are much smaller. Therefore in the remainder of the chapter, we will neglect cross terms. Also we will restrict ourselves to the case without an external magnetic field, hence the subindex $m$ in Eqs. (14) and (15) may be dropped.

The integral equations may be studied using an iteration method. If $N$ is large, we expect a rapid convergence. ${ }^{11,13,17} \mathrm{We}$ find that at zero temperature there exists a common solution $E$ for Eqs. (14) and (15), and this solution is 
more negative than the other solutions for either Eq. (14) or Eq. (15). This common solution $E$ is the ground state energy of the system. This is consistent with the result by Inagaki ${ }^{18}$ who applied Yosida-Yoshimori theory to treat the ground state of the system. This is also consistent with the result of analytic study of the integral equations. There may exist other solutions for Eq. (14) or Eq. (15), depending on the parameters of the system, such as $N \Delta / D, \epsilon_{f} / D$ and $N$.

Now consider the $f$-electron function defined as

$$
\begin{aligned}
G_{m m}\left(\tau_{a}, \tau_{b}\right)= & -T_{r}\left\{e^{-B H_{i}} T_{\tau}\right. \\
& \left.\times\left[X_{m}\left(\tau_{a}\right) X_{m}^{+}\left(\tau_{b}\right) e^{--\delta_{0}^{(B} d \tau H_{1}(\tau)}\right]\right\} / Z .
\end{aligned}
$$

It is easy to show that the Fourier transform of the Green function $G_{m m}\left(i \omega_{n}\right)$ relates to the partition function $Z$ in the following simple way:

$G_{m m}\left(i \omega_{n}\right)=-\left.\frac{1}{\beta} \frac{\partial}{\partial\left(f_{k \sigma}\left|V_{k \sigma m}\right|^{2}\right)} \ln Z\right|_{\epsilon_{k \sigma}+i \omega_{n}}$,

where $\epsilon_{k \sigma} \rightarrow i \omega_{n}$ means that we replace $\epsilon_{k \sigma}$ by $i \omega_{n}$ after taking the derivative. Thus $G_{m m}\left(i \omega_{n}\right)$ may be expressed diagrammatically, if we cut a conduction band electron line and use $i \omega_{n}$ to substitute for it. Disregarding cross terms, we can sum up the diagrams and obtain ${ }^{15}$

$$
\begin{aligned}
G_{m m}\left(i \omega_{n}\right) & =\frac{1}{2 \pi i Z /\left(Z_{0}\right)_{c}} \int d z \\
\times & \frac{e^{-\beta z}}{[z-S(z)]\left[z+i \omega_{n}-\epsilon_{f}-T\left(z+i \omega_{n}-\epsilon_{f}\right)\right]},
\end{aligned}
$$

which is an extension of the noninteracting Green function

$$
G_{m m}^{0}\left(i \omega_{n}\right)=\frac{n_{0}}{2 \pi i} \int d z \frac{e^{-\beta z}}{z\left(z+i \omega_{n}-\epsilon_{f}\right)} .
$$

Taking the analytic continuation of Eq. (18), one can obtain a retarded Green function $G_{m m}(\omega-i \delta)$, the imaginary part of which gives the spectral function

$$
\begin{aligned}
R_{m}(\omega)= & \frac{1}{\pi^{2} Z /\left(Z_{0}\right)_{c}} \int d x e^{-\beta x}\left(1+e^{-\beta(\omega)}\right) \\
& \times \operatorname{Im}\left(\frac{1}{x-i \delta-S(x-i \delta)}\right) \\
& \times \operatorname{Im}\left(\frac{1}{x+\omega-\epsilon_{f}-i \delta-T\left(x+\omega-\epsilon_{f}-\mathrm{i} \delta\right)}\right) .
\end{aligned}
$$

If $\Delta=0$, Eq. (20) becomes

$$
R_{m}^{0}(\omega)=\delta\left(\omega-\epsilon_{f}\right)\left(n_{0}+n_{m}\right),
$$

which is just the spectral function for a noninteracting system. The structure of the spectral function in Eq. (20) is a product of two imaginary parts, $\{\operatorname{Im}[x-S(x-i \delta)]\}^{-1}$ and $\left\{\operatorname{Im}\left[x+\omega-\epsilon_{f}-T\left(x+\omega-\epsilon_{f}-i \delta\right)\right]\right\}^{-1}$, which are the components of the effective density of states. Equation (20) reflects hopping between the $f$ electron and the $f$ hole.

The expectation value of the $f$ electron occupation number $\left\langle n_{f}\right\rangle=\Sigma_{m} \int R_{m}(\omega) f(\omega) d \omega$ can be derived from Eq. (20):

$$
\begin{aligned}
\left\langle n_{f}\right\rangle= & \sum_{m} \frac{1}{\pi Z /\left(Z_{0}\right)_{c}} \int d x e^{-\beta x} \\
& \times \operatorname{Im} \frac{1}{x-\epsilon_{f}-i \delta-T\left(x-\epsilon_{f}-i \delta\right)},
\end{aligned}
$$

and the $f$-hole occupation number $\left\langle 1-n_{f}\right\rangle=\int R_{m}$ $\times[1-f(\omega)] d \omega$ is

$$
\left\langle 1-n_{f}\right\rangle=\frac{1}{\pi Z /\left(Z_{0}\right)_{c}} \int d x e^{-\beta x} \operatorname{Im} \frac{1}{x-i \delta-S(x-i \delta)} .
$$

This shows that the normalization is satisfied for Eqs. (18) and (20), although the crossing diagrams are neglected.

We have presented a new diagrammatic method for the degenerate Anderson model. This technique avoids the complication of regularization required in the old method proposed by Keiter and Kimball. ${ }^{9}$ The partition function is formulated exactly. The $f$-electron Green function and spectral function can be derived from the partition function. Simple forms of Green function and spectral function are obtained if we neglect crossing diagrams, but normalization is still satisfied. This theory enables us to calculate physical quantities. The results will be published elsewhere.

We like to acknowledge that same results for Green function and spectral function are derived in different ways simultaneously by P. Coleman ${ }^{19}$ and Y. Kuramoto. ${ }^{20}$

\footnotetext{
Valence Fluctuation in Solids, edited by L. M. Falicov, W. Hanke, and M. B. Maple (North-Holland, Amsterdam, 1981).

"Proceedings of the International Conference on Valence Instabilities, edited by P. Wachter (North-Holland, Amsterdam, 1982).

${ }^{3}$ B. Coqblin and J. R. Schrieffer, Phys. Rev. 185, 847 (1969); J. H. Jefferson and K. W. H. Stevens, J. Phys. C 9, 2151 (1976).

${ }^{4}$ P. W. Anderson, Phys. Rev. 126, 41 (1961).

${ }^{5}$ For example, O. Gunnarsson and K. Schoenhammer (preprint) (1983)

'P. B. Wiegmann, Phys. Lett. 80A, 163 (1980); A. M. Tsvelick and P. B. Wiegmann, Phys. Lett. 89, 368 (1982); N. Andrei, Phys. Rev. Lett. 45, 379 (1980).

P. Schlottmann, Z. Phys. B 49, 109 (1982)

${ }^{8}$ H. R. Krishnamurthy, J. W. Wilkins, and K. G. Wilson, Phys. Rev. B 21. 1044 (1980).

${ }^{\circ}$ H. Keiter and J. C. Kimball, Int. J. Magn. 1, 233 (1971).

${ }^{16)}$ R. Balian and C. De Dominicis, Ann. Phys. (N. Y.) 62, 229 (1971).

${ }^{\prime \prime}$ T. V. Ramakrishnan, Ref. 1, p. 13.

${ }^{12}$ A. Bringer and H. Lustfeld, Z. Phys. B 28, 213 (1977).

${ }^{1.3}$ F. C. Zhang and T. K. Lee, Phys. Rev. B 28, 33 (1983).

${ }^{14} \mathrm{G}$. Czycholl, H. Keiter and E. Niebur, Ref. 2.

${ }^{15}$ H. Keiter and G. Czycholl (preprint) (1983).

${ }^{1}$ T. K. Lee and F. C. Zhang (to be published).

${ }^{17}$ P. W. Anderson, Ref. 1, p. 451.

${ }^{18}$ S. Inagaki, Progs. Theor. Phys. 62, 1441 (1979).

${ }^{19} \mathrm{P}$. Coleman (preprint) (1983).

${ }^{20}$ Y. Kuramoto (preprint) (1983)
} 